Review Article

\title{
Effect of food on causation and prevention of gastric
} cancer

\begin{abstract}
Stomach cancer is the fourth leading cause of cancer related death in both males and females worldwide. ${ }^{1}$ Food preservatives and salt are responsible for causing gastric cancer. Fresh fruits, vegetables, carotene, Vitamin C, Calcium, Garlic, Mushrooms, Citrus fruits, Milk and milk products prevent from stomach cancer. The most effective way to prevent cancer is to avoid food that cause cancer and increase the consumption of food that prevent from cancer. The knowledge of the factors that cause and prevent from gastric cancer will be helpful in decreasing the incidence of gastric cancer.
\end{abstract}

Keywords: stomach cancer, salt, food preservatives, garlic, vegetables, allicin, selenium, anticancer, antioxidant
Volume 8 Issue 5 - 2017

Aqsa lqbal

Department of Physiology and Biophysics, Center of

Cardiovascular Research, University of Illinois, USA

Correspondence: Aqsa Iqbal, University of Illinois at Chicago, Illinois, USA, Email aiqbal20@uic.edu

Received: August 15, 2017 | Published: September 20, 2017
Abbreviations: TNF, tumor necrosis factor; IL, interleukin; GSH: glutathione; GST: glutathione-s-transferase; GPx, glutathione peroxidase; MCP: monocyte chemoattractant protein; Cag-A: cytotoxic-associated gene A; SOD, Superoxide dismutase; DNA: deoxyribonucleic acid

\section{Introduction}

Stomach cancer is the $5^{\text {th }}$ most common cancer in the world after lung, breast, colorectal and prostate. According to WHO, gastric cancer is the $4^{\text {th }}$ leading cause of cancer related death worldwide with Lung, Liver, and Colorectal cancers are the $1^{\text {st }}, 2^{\text {nd }}$ and $3^{\text {rd }}$ respectively. ${ }^{1,2}$ According to American cancer society, the average age of people diagnosed with stomach cancer is 69 . The risk is twice as higher in men than women. Overall, the risk of stomach cancer is declining due to decrease in Helicobacter Pylori infection or improved treatment options and the use of refrigeration to preserve foods. Globally, stomach non-cardiac cancer is more common than cardiac cancer. Stomach cancer is more common in Japan, China, Southern and Eastern Europe, and South and Central America worldwide.

\section{Types of stomach sancers}

a. Adenocarcinoma, (most common type of stomach cancer and classified histologically by Lauren into Intestinal and diffuse type). ${ }^{3}$

b. Lymphoma

c. Gastrointestinal stromal tumor

d. Carcinoid tumor

e. Squamous cell carcinoma etc.

\section{Risk factor of stomach cancer}

a. H. Pylori infection ${ }^{4}$ (Classified as Class I Carcinogen by World Health Organization and cause gastric cancer directly by epigenetic effect and indirectly through inflammatory response). ${ }^{5,6}$

b. Smoking ${ }^{4}$ (especially waterpipe tobacco smoking- Smoking flavored tobacco through a long pipe attached to a water bowl). ${ }^{7}$ c. Blood group A. ${ }^{7}$

d. Proton pump inhibitor (Cause hypergastrinemia which leads to gastrointestinal neoplasia). ${ }^{7,8}$

e. Pernicious anemia. ${ }^{7}$

f. Organ transplant recipient. ${ }^{7}$

g. Alcohol (production of prostaglandins, lipid peroxidation and the generation of oxygen radical species leads to gastric cancer) ${ }^{4}$

h. Overweight and obesity $(\mathrm{BMI}>25)$ (Obesity is associated with low-grade chronic inflammatory state, with increased production of TNF, IL-6, MCP-1 and C- reactive protein. Chronic inflammation promotes gastric cancer). ${ }^{9}$

i. Epstein-Bar virus $(\mathrm{EBV})^{4}$ - prefer non-antral location in stomach. EBV gene incorporates into human genes and cause gastric cancer.
i. Positive family history. ${ }^{7}$
ii. Low socioeconomic status. ${ }^{4}$
iii. Gastro esophageal reflux. ${ }^{7}$

\section{Purpose}

a. The purpose of this article is

b. Summarize different foods that cause stomach cancer in different countries so that those foods can be avoided

c. Summarize different foods that prevent stomach cancer.

d. Give insight on new research topics (Table 1).

\section{Salt and gastric cancer ${ }^{10-12}$}

Salt has long been known for its adverse health effects, including Meniere's disease, worsening of renal diseases, Hypertension, triggering an asthma attack, osteoporosis, exacerbation of fluid retention, renal calculi etc. Incidence of gastric cancer is higher in countries where traditional diet contain large amount of salt and salt preserves food. This article explains the mechanisms behind gastric cancer causation by salt and salt preserved food. 
Table I List of unusual foods cause stomach cancer

Sa-um (Fermented pork fat) ${ }^{29}$
Sour pancakes (Fermented local food in China) $)^{30}$
Bekang (Fermented soya bean) $)^{30}$
Soda (Alkali)
Salt preserved meat
Cooked rice with beans ${ }^{31}$
Pallock soup (Korea) ${ }^{31}$
Charcoal grilled beef (Korea) ${ }^{31}$
Kkakduki (A kind of kimchi prepared with
salted radish and red pepper etc) (Korea) $)^{31}$
Dongchimi (A kind of kimchi prepared with radish
and a large quantity of salt water) (Korea) ${ }^{31}$
Cooked spinach (Korea) ${ }^{31}$

\section{Mechanism ${ }^{12}$}

a. The high salt intake strips the lining of stomach, leading to inflammation and atrophy. Such damage may make infection with $H$. pylori more likely or may exacerbate the infection by enhancing cagA expression ( $\mathrm{p} 53$, which is responsible for apoptosis of cells, is inhibited by cag A). cagA is a virulence factor of $H$. pylori. High level of salt also synergistically augments the expression of proinflammatory enzymes (nitric oxide synthase and cyclooxygenase-2) and cytokines in gastric mucosa with $H$. pylori infection. A statistically significant decrease in gastric cancer has been seen after 2 weeks antibiotic treatment of Helicobacter pylori. H. Pylori treatment with amoxicillin and omeprazole had positive effects on severity of precancerous lesions and decrease the prevalence of precancerous lesion. ${ }^{4,6,13,14}$

b. High level of sodium chloride damage the walls of stomach, cause cell death and induce regenerative cell proliferation resulting in inflammation. Cell proliferation boosts the effect of food-derived carcinogen. ${ }^{10}$

c. Mutagens are produced when salt acts synergistically with nitrates. Mutagens cause gastric cancer.

d. Micronutrient which protect from gastric cancer are lower in salt preserved foods.

\section{Strategies to decrease salt intake around the globe. ${ }^{15-17}$}

Designing and implementing salt reduction strategies would be very helpful in reducing the risk of gastric cancer. WHO advises salt reduction as one of the most economical and efficient approach to prevent non-communicable diseases. Some of the salt reducing strategies used around the globe is:

a. Consumer education

b. Food industry engagement to reformulate products. Following is the list of targeted foods for reformulations

Bread is the most targeted food for reformulation

Bakery products,

Processed meats,

Dairy products,

Sauces and convenience meals.
c. Front of pack labeling
d. Intervention in public institution settings (such as schools, hospitals and the workplace)
e. Taxation of high sodium products

\section{Food preservatives and stomach cancer. ${ }^{18-22}$}

Food preservatives, which are used to prevent the spoilage of food, have been in use since ancient times. Artificial sweeteners, monosodium glutamate, sodium sulfite and sodium nitrate/sodium nitrite are some of the examples of food preservatives. Among them, sodium nitrates and nitrites are associated with elevated risk of gastric cancer. Sodium nitrates and nitrites leads to the formation of N- nitro compounds, which are known as human and animal carcinogen. NNitroso compounds, which are formed from nitrates and nitrites, are main cause responsible for causing cancer due to food preservatives. $\mathrm{N}$ - nitroso compounds consist of three main chemical groups ${ }^{4}$.

A. N-Nitrosamines (DMN)

B. Nitrosamides (MNU)

\section{Nitrosaminidine (MNNG)}

There are two sources of $\mathrm{N}$ - nitro compounds in our body.

a. Exogenous - Tobacco, drugs, diet, and occupational environment are the exogenous source of N- nitroso compounds. Foods that contain preformed nitrosamine are smoked preserved foods, cured meat products, salty preserved foods, and food dried by additives such as malt in the production of beer and whiskey

b. Endogenous - Processed meat, which include bacon, sausage, hot dogs, salami, corned beef etc., contain large amount of nitrates and nitrite. Endogenously, N - Nitroso compounds are produced

c. when nitrates and nitrites react with amino acids in stomach. Haem iron in processed meat leads to endogenous production of $\mathrm{N}$ - nitro compounds. Haem iron also increase the progress of $H$. pylori infection by causing DNA damage and oxidative stress. Ascorbic acid decreased the burden of endogenously formed NNitro compounds.

\section{Mechanism}

A.N- Nitroso compounds are carcinogenic, mutagenic and teratogenic. They cause carcinogenesis by causing mutations in genes.

B. N- NItroso compounds are metabolized into a reactive intermediates (a methyl- Carbonium ion) which reacts with various cellular macromolecules. According to one study, alkyl group, reactive intermediates, alkylate the O6- Position of guanine in DNA, which is involved in the normal Watson Crick base pairing, resulting in carcinogenesis (Figure 1).

\section{Protective properties of garlic ${ }^{23-26}$}

Garlic (Allium sativum) is one of the best-selling herbal products in the market due to its beneficial effects on health. Garlic has antibacterial, antihypertensive and antibiotic properties. It improves immune mechanism of our body and helpful in the treatment of cardiovascular diseases and cancers. Vegetables in the allium group, that is, garlic, onions and Chinese chives demonstrate negative association with gastric cancer in a dose-response pattern (Figure 2). 


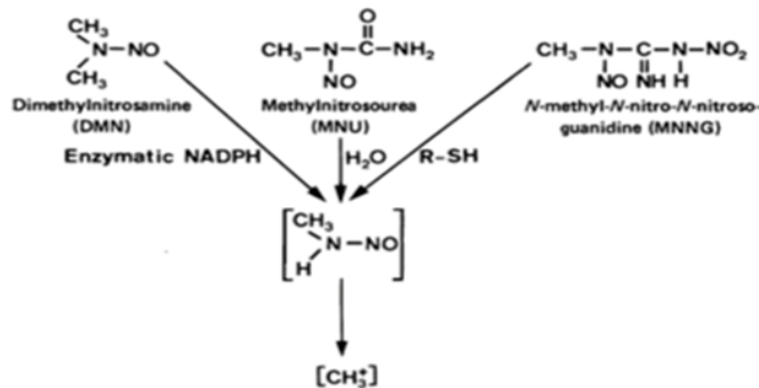

Figure I Reactive intermediate formation from N- Nitroso compounds. ${ }^{22}$

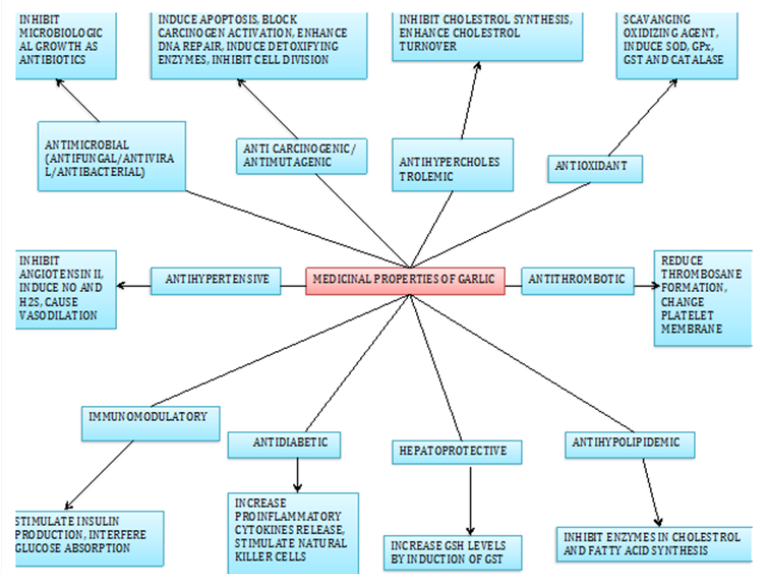

Figure 2 Medicinal properties of garlic. ${ }^{27}$

\section{Mechanism $^{27}$}

Garlic contains more then 2000 biologically active substances. Some of these substances act as antioxidant and its sulfur compounds have anti-tumor properties The anti-carcinogenic effects of garlic is attributable to organosulfur compounds such as alliin, alliinase, allicin, S-allyl cysteine (SAC),diallyldisulphide (DADS), diallyltrisulphide (DATS) and methylallyltrisuphide, which are highly effective in affording protection against cancer. Protein fraction from garlic prevent tumor growth by infiltrating CD8 $(+) \mathrm{T}$ - cell in tumor site. Diallyl trisulfide (DATS) have antioxidant, anti-inflammatory, and anti-carcinogenic properties by down regulating AKT1/TGFB-activated Kinase mediated MAPK and nuclear factor kappalight-chain-enhancers of activated B cells (NF-kB) pathways. Allyl compounds and isothiocyanates act as histone deacetylases inhibitors (HDACi). They activated epigenetically silenced genes in cancer cells, causing cell cycle arrested and apoptosis. Allicin and methyl linoleate are 2 phytochemical present in garlic. These chemicals induce enzymatic detoxification system including mixed function oxidase system and glutathione-S-transferase. Allicin inhibit growth of $\mathrm{H}$. pylori, prevents the progression of tumor cells, arrest cell cycle at G2/M phase, and induce apoptosis. Garlic is a seleniferous plant, accumulating selenium from the soil against a concentration gradient. Selenium has many anticancer actions, particularly in control of genes involved in carcinogenesis.

Increased consumption of garlic has been associated with a lower rate of progression for chronic atrophic gastritis, and also for intestinal metaplasia/gastric metaplasia. Garlic has antibiotic properties and Individuals who consume garlic are at low risk of H. Pylori infection.
Oxidative damage plays a role in gastric carcinogenesis, and is inhibited by anti-oxidants such as those found in garlic

\section{Gastric cancer prevention}

Prevention is critical to reduce gastric cancer mortality

i. Screen and treat Helicobacter pylori infection in high risk areas and first degree relatives of gastric cancer patients and in those having high risk of developing gastric cancer. ${ }^{28}$

ii. Increase intake of following foods

iii. Reduce consumption of salt and salt- preserved foods, smoked foods and red meat.

iv. Smoking cessation

v. Reduction of alcohol intake

vi. Increase physical activity

vii. Diagnose and treat gastric precancerous lesions by endoscopic screening. ${ }^{4}$

viii. Biomarker screening (Non -invasive, Low pepsinogen 1 or pepsinogen 1 /pepsinogen 2 ratio and negative H.pylori antibodies suggest the highest risk of gastric cancer). ${ }^{4}$ (Table 2).

Table 2 List of foods prevent stomach cancer

Fresh fruits and vegetables ${ }^{4}$

Carotene,Vitamin $\mathrm{C}$ (enhance mucosal immune response, neutralizing free radicles,

decrease formation of $\mathrm{N}$ - nitro compounds and influence growth of $H$. pylori),

Vitamin E, and Calcium ${ }^{30,32}$

Garlic $^{31}$

Mushrooms 2,31

Citrus fruits. ${ }^{2,31}$

Milk and milk products ${ }^{31}$

Pulses and foods containing selenium ${ }^{26}$

Aqueous rice extract

\section{Discussion}

Salt, food preservatives and $H$. pylori can cause gastric cancer independently and in combination with each other. An association has been found between salt intake and emergence of gastric cancer. Effect of salt on gastric cancer causation is a well-established fact. We need to find out the quantity and time period of the salt takes to cause gastric cancer. Although, studies have been done with using different amount of salt on animals but more work needs to be done on amount of salt that promotes the development of stomach cancer. Many countries use strategies to reduce the amount of salt intake but in order to successfully reduce the risk of gastric cancer; carcinogenic quantity of salt should be calculated. Salt is an essential component of diet and it is difficult to eliminate its use but if the carcinogenic quantity of salt is determined, more efficient strategies can be developed.

Nitrates and nitrates which are especially used in processed food cause gastric cancer by $\mathrm{N}$ - Nitroso compound formation. Reactive intermediates of N- Nitroso compounds react with proteins and DNA in cells and cause cancer. Nitrates and nitrites have long been the cause 
of cancers but no antidote has been developed to prevent damage caused by them. As the bacteria develop resistance to antibiotics in the same way more research should be done to acquire resistance from carcinogens. Garlic contains many substances that prevent from cancers. These substances have antibiotic, anti-inflammatory, and antioxidant properties. Garlic should be used more to decrease the risk of gastric cancer. ${ }^{33}$.

\section{Conflicts of interest}

There is no conflict of interest.

\section{Acknowledgements}

None.

\section{Funding}

None.

\section{References}

1. WHO. (2017) International agency for research on cancer. World health Organization.

2. World cancer research fund international. Diet, Nutrition, physical activity and stomach cancer. 2016.

3. Lauren P. The Two Histological Main Types of Gastric Carcinoma: Diffuse and So- Called Intestinal- Type Carcinoma. An Attempt at a Histolo- Clinical Classification. Acta Pathol Microbiol Scand. 1965;64(1):31-49.

4. Xiao Jiao Cheng, Jia Cheng Lin, Shui Ping Tu. Etiology and Prevention of Gastric Cancer. Karger. 2016;3(1):25-36.

5. Ishaq S, Nunn L. Helicobacter pylori and Gastric Cancer. Gastroenterol Hepatol Bed Bench. 2015;8(Suppl1):S6-S14.

6. Karimi P, Islami F, Anandasabapathy S, et al. Gastric cancer: descriptive epidemiology, risk factors, screening, and prevention. Cancer Epidemiol Biomarkers Prev. 2014;3:700-713.

7. Mamtani R, Cheema S, Sheikh J, et al. Cancer risk in waterpipe smoker: a meta-analysis. Int J Public Health. 2016;62(1):73-83.

8. Poulsen AH, Christensen S, McLaughlin JK, et al. Proton Pump Inhibitors and Risk Of Gastric Cancer: a population- based cohort study. Br J Cancer. 2009;100(9):1503-1507.

9. Lin XJ, Wang CP, Liu XD, et al. Body mass index and risk of gastric cancer: a meta-analysis. Jpn J Clin Oncol. 2014;44(9):783-791.

10. Furihata $\mathrm{C}$, Ishida $\mathrm{S}$, Ohta $\mathrm{H}$, et al. Ctotoxicity of $\mathrm{NaCl}$, a stomach tumor promotor, and prevention by rice extract in stomach mucosa of F344 rats. Cancer Detect Prev. 1996;20(3):193-198.

11. Kim HJ, Chang WK, Kim MK, et al. Dietary factors and gastric cancer in Korea: a case-control study. Int J Cancer. 2002;97(4):531-535.

12. S Tsugane, S Sasazuki, M Kobayashi, et al. Salt and salted food intake and subsequent risk of gastric cancer among middle-aged Japanese men and women. Br J Cancer. 2004;90(1):128-134.

13. Buti L, Spooner E, Van der Veen AG, et al. Helicobacter pylori cytotoxin-associated gene $\mathrm{A}(\mathrm{CagA})$ subverts the apoptosis-stimulating protein of p53 (ASPP2) tumor suppressor pathway of the host. Proc Natl Acad Sci USA. 2011;108 (22):9238-9243.
14. Toyoda T, Tsukamoto T, Hirano N, et al. Synergistic upregulation of inducible nitric oxide synthase and cyclooxygenase -2 in gastric mucosa of Mongolian gerbils by a high-salt diet and Helicobacter pylori infection. Histol Histopatho. 2008; 23(5):593-599.

15. WHO. Global status report on noncommunicable diseases. World Health Organization. 2010.

16. Kathy Trieu, Bruce Neal, Corinna Hawkes, et al. Salt Reduction Initiatives around the World - A Systematic Review of Progress towards the Global Target. PLoS One. 2015;10(7): e0130247.

17. World Health Organization. Salt reduction fact sheet. 2016.

18. Loh YH, Jakszyn P, Luben RN, et al. N-Nitroso compounds and cancer incidence: the European Prospective Investigation into Cancer and Nutrition (EPIC)-Norfolk Study. Am J Clin Nutr. 2011;93(5):10531061.

19. P Jakszyn, CA González. Nitrosamine and related food intake and gastric and oesophageal cancer risk: A systematic review of the epidemiological evidence. World J Gastroenterol. 2006;12(27):4296-4303.

20. Sun J1, Aoki K, Wang W, et al. Sodium nitrite-induced cytotoxicity in cultured human gastric epithelial cells. Toxicol In Vitro. 2006; 20(7):1133-1138.

21. R Montesano, J Hall. Nitrosamine metabolism and carcinogenesis. Mutation, Cancer, and Malformation. 1984;31:447-464.

22. P F Swann. Carcinogenic risk from nitrite, nitrate and $\mathrm{N}$-nitrosamines in food. Proc R Soc Med. 1977; 70(2):113-115.

23. Garcia Martin R, Matia Cubillo A. Influence of diet in primary prevention of gastric cancer, in patients infected with Helicobacter pylori. Rev Enferm. 2016;39(5):33-38.

24. Majewski M. Allium sativum: facts and myths regarding human health. Rocz Panstw Zaki Hig. 2014;65(1):1-8.

25. Luo R, Fang D, Hang H, et al. The mechanism in gastric cancer chemoprevention by allicin. Anticancer Agents Med Chem. 2016;16(7):802-809.

26. Pandrani A. Cancer chemoprevention by garlic - A review. Hereditary genet. 2015; 4(2):1-7.

27. Gao C, Jiang X, Wang H, et al. Drug metabolism and pharmacokinetics of organosulfur compounds from garlic. J Drug Metab Toxicol. 2013;4(5):1-10.

28. You WC, Brown LM, Zhang L, et al. Randomized double-blind factorial trial of three treatments to reduce the prevalence of precancerous gastric lesions. J Natl Cancer Inst. 2006;98(14):974-983.

29. Phukan RK, Narain K, Zomawia E, et al. Dietry habits and stomach cancer in Mizoram. India J Gastroenterol. 2006;41(5):418-424.

30. You WC, Li JY, Zhang L, et al. Etiology and prevention of gastric cancer: a population study in a high risk area of China. China J Dig Dis. 2005;6(4):149-154.

31. Strand M. Salt and Cancer. Acta Med Croatica. 2010;64(2):159-161.

32. Ma JL, Zhang L, Brown LM, et al. Fifteen-year effects of Helicobacter pylori, garlic, and vitamin treatments on gastric cancer incidence and mortality. J Natl Cancer Inst. 2012;104(6):488-492.

33. Cancer Research UK. Stomach Cancer Risk factors. 